\title{
Chest Wall Reconstruction with Precontoured Locking Plate Proof of Concept
}

\author{
Elmer Lodder, Maarten van der Elst
}

Reinier de Graaf Hospital, Reinier de Graafweg 3-11, Delft, Netherlands.

Email: elmerlodder@hotmail.com

Received April 21 ${ }^{\text {st }}, 2010$; revised May 21 $1^{\text {st }}, 2010$; accepted June $15^{\text {th }}, 2010$.

\begin{abstract}
Complications after chest wall resection are common and are reported to occur in approximately $40 \%$ of patients. The most frequent complications are respiratory or wound complications. Restoring rib continuity after a resection is likely to prevent respiratory complications. However many patients remain painful after a reconstruction. This article describes a new technique using a titanium alloy precontoured locking plate and locking screws to reconstruct the chest wall after resection of the $7^{\text {th }}$ rib on the left.
\end{abstract}

Keywords: Chest Wall Resection and Reconstruction

\section{Introduction}

Complications after chest wall resection are common and are reported to occur in $37 \%$ to $46 \%$ of patients. Respiratory complications are most frequent, ranging from $20 \%$ to $24 \%$ [1] Wound infections occur in $5 \%$ [1] to $11 \%$ [2]. Furthermore many patients experience chronic pain. Restoring rib continuity after a resection is likely to prevent respiratory complications. Possible ways to reconstruct after chest wall resection are approximating adjacent ribs for small resections, or using non-rigid reconstructions like a polypropylene or vicryl mesh, or rigid reconstructions like polypropylene mesh/methylmethacrylate composite. This article describes a new technique by using a titanium alloy precontoured locking plate and locking screws to reconstruct the chest wall.

\section{Case Report}

Three years after a nephrectomy on the right side, because of a clearcell renal carcinoma, a 73-year-old female was diagnosed with a solitary metastasis in the left $7^{\text {th }}$ rib. This metastasis was discovered on a CT-scan (Figure 1), that was made because of recurring urinary tract infections and pain on her left thorax side. The patient gave informed consent for publication of this article.

The skin and fat was incised over the $7^{\text {th }}$ rib on the left, with the patient lying on her right sight. The latissimus dorsi and serratus anterior muscles were partially opened and the tumor was exposed. The diameter of the tumor was approximately $6 \mathrm{~cm}$ and invaded the pleura. The rib was cut in healthy bone tissue on either side of the tumor and the tumor was excised including pleura. The continuity of the rib was restored by using a titanium precontoured locking plate and locking screws (Figure 2). The $6^{\text {th }}$ and $8^{\text {th }}$ rib were approximated using a double Vicryl. A thoraxdrain was left because of the pleural defect. The muscles were approximated using PDS, then subcutis and skin were closed.

The patient was extubated immediately after the operation. Painmedication consisted of a epidural catheter for 3 days. The thoraxdrain was removed 3 days after the operation and she left the hospital 4 days later in good condition.

At 2-months follow-up she had no pain, good thorax excursions and had picked up her daily activities.

\section{Conclusions}

Titanium is a strong, light and nonferromagnetic material with low density, causing little interference on computed tomography. The precountered plate and locking screws used in this case provided a stable reconstruction. The patient had no respiratory or wound complications, made a quick and full recovery, and reported no pain. It is therefore an effective way of restoring rib continuity after a resection. It should be considered as a proof of concept. Further investigation is required to proof it is also effective for multiple rib resections. 


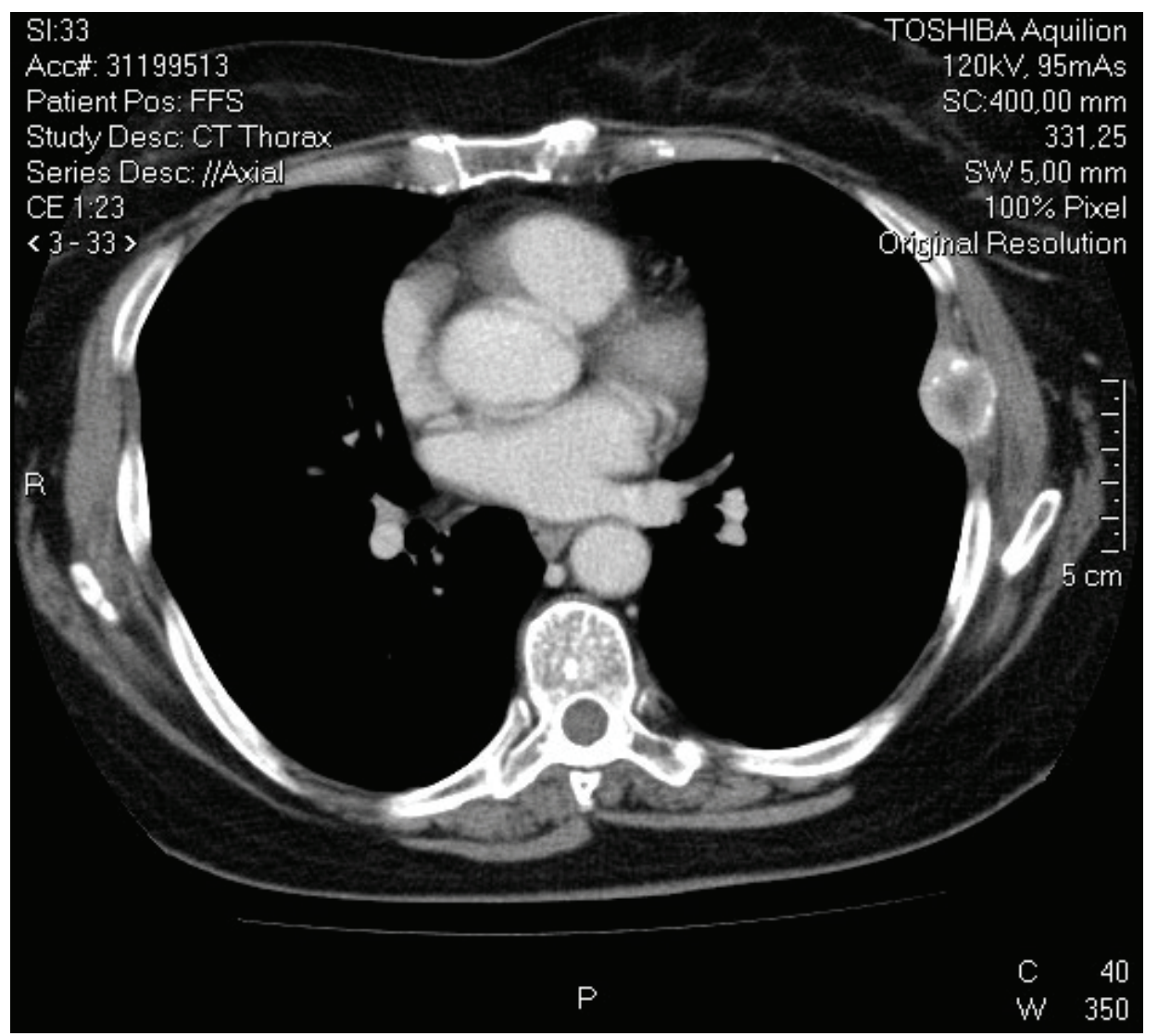

Figure 1. CT-scan showing metastasis in the $7^{\text {th }}$ rib left.

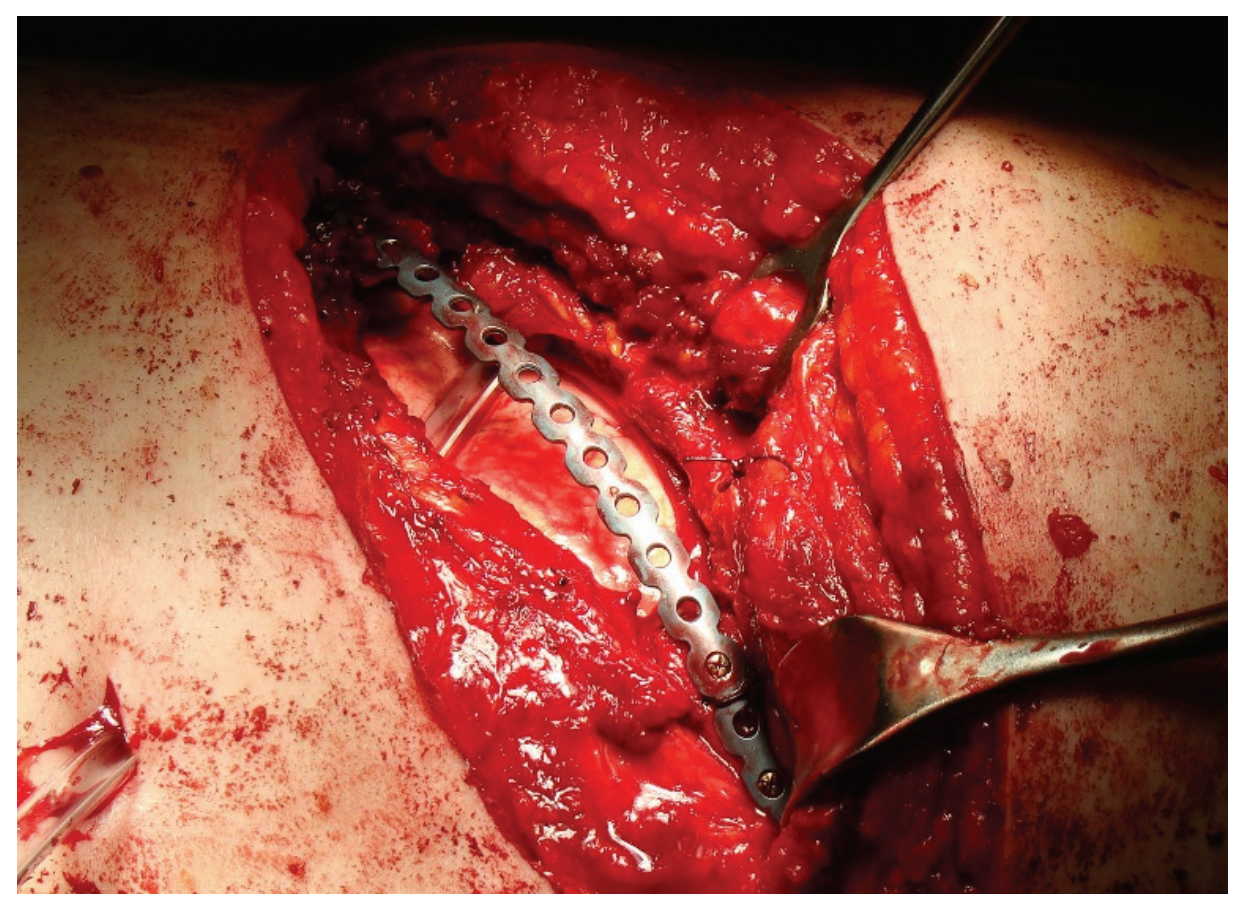

Figure 2. rib reconstruction using a titanium precontoured locking plate and locking screws. 


\section{REFERENCES}

[1] M. J. Weyant, M. S. Bains, E. Venkatraman, R. J. Downey, B. J. Park, R. M. Flores, N. Rizk and V. W. Rusch, "Results of Chest Wall Resection and Reconstruction with and without Rigid Prosthes," The Annals of Thoracic Surgery, Vol. 81, No. 1, 2006, pp. 279-285.
[2] T. E. Lans, C. van der Pol, M. W. Wouters, P. I. M. Schmitz and A. N. van Geel, "Complications in Wound Healing after Chest Wall Resection in Cancer Patients - A Multivariate Analysis of 220 Patients," Journal of Thoracic Oncology, Vol. 4, No. 5, 2009, pp. 639-643. 